\title{
Wrong papers in mathematics I. Paper by Cakoni F., Colton D., Monk P. in "Inverse Problems" and subsequent papers
}

\author{
P.A. Krutitskii \\ KIAM, Miusskaya Sq. 4, Moscow 125047, Russia \\ Email: biem@mail.ru
}

\begin{abstract}
The long series of mistaken papers published in the mathematical journals with very high impact factor during 11 years is discussed. The following journals published these mistaken papers: Inverse Problems (1), Nonlinear Analysis (1), Boundary Value Problems (1), Journal of Differential Equations (1), Journal of Mathematical Physiscs (2), Mathematical Methods in the Applied Sciences (2). The number in brackets shows the number of published wrong papers involved in the discussion. This series of wrong papers has been generated by a mistake in the paper:

Cakoni F., Colton D. and Monk P. The direct and inverse scattering problems for partially coated obstacles. Inverse Problems, 2001, v. 17, pp. $1997-2015$.
\end{abstract}

Keywords: High impact factor, mistaken papers, adjoint operator, boundary value problems, integral equations.

\section{Introduction}

Mathematicians read journals with high impact factor and trust papers published there. They study morden methods presented in these journals to use them in their research. Sometimes such journals publish wrong papers, then wrong methods are spreading in mathematics communitiy. The only way to prevent spreading of such wrong results is to publish "Letters to Editors", where a reader may inform other readers about mistakes found in the journal. However journals with high impact do not like to publish letters of readers about mistakes, since they think that they may preserve good reputation without such letters only. In this case readers should publish their observations about mistaken papers in other journals, to prevent spreading of wrong results in mathematical community. Reputation of journals is not so important thing as spreading of wrong mathematics. In this note we discuss a wrong paper in a mathematical journal with very high impact and consequences of its publication. 


\section{Paper in "Inverse Problems" published in 2001}

One of basic results of the paper "The direct and inverse scattering problems for partially coated obstacles" by Cakoni F., Colton D. and Monk P. published in Inverse Problems, 2001, v. 17, pp. $1997-2015$, is a solvability of a certain boundary value problem, and this result is formulated in Theorem 2.5. However the proof of Theorem 2.5 is wrong. The proof uses relation (29) and a subsequent relation that are based on the assumption that the operator $K^{\prime}$ is adjoint to the operator $K$, where (see p.2001)

$$
\begin{aligned}
& K \psi=2 \int_{\Gamma} \psi(y) \frac{\partial \Phi(x, y)}{\partial \nu_{y}} d s_{y}, \quad K^{\prime} \psi=2 \int_{\Gamma} \psi(y) \frac{\partial \Phi(x, y)}{\partial \nu_{x}} d s_{y} \\
& \Phi(x, y)=\frac{i}{4} H_{0}^{(1)}(k|x-y|), \quad k>0
\end{aligned}
$$

and $H_{0}^{(1)}(z)$ is a Hankel function. However this assumption is wrong, the operator $K^{\prime}$ is not adjoint to the operator $K$, and the relation (29) and the subsequent relation are mistaken. The correct adjoint operator to the operator $K$ was presented, for example, in [1, p. 162], and it is defined by

$$
A \psi=2 \int_{\Gamma} \psi(y) \frac{\partial \overline{\Phi(x, y)}}{\partial \nu_{x}} d s_{y}
$$

where overline means complex conjunction. Notice that $A \neq K^{\prime}$, since

$$
\Phi(x, y) \not \equiv \overline{\Phi(x, y)}=-\frac{i}{4} \overline{H_{0}^{(1)}(k|x-y|)}=-\frac{i}{4} H_{0}^{(2)}(k|x-y|),
$$

where relations for Hankel functions from [2]

$$
H_{0}^{(1)}(z)=J_{0}(z)+i Y_{0}(z), \quad H_{0}^{(2)}(z)=J_{0}(z)-i Y_{0}(z)
$$

are used, and $J_{0}(z)$ and $Y_{0}(z)$ are Bessel functions of the first and second kind. Indeed, one can check that

$$
\Phi(x, y)-\overline{\Phi(x, y)}=\frac{i}{4}\left(H_{0}^{(1)}(k|x-y|)+H_{0}^{(2)}(k|x-y|)\right)=\frac{i}{2} J_{0}(k|x-y|) \not \equiv 0 .
$$

Notice that scalar product $(a, b)$ means integral of $a \cdot \bar{b}$ in the paper by Cakoni F., Colton D. and Monk P.

The "World of Science" database screens 39 references to this mistaken paper at the moment. It would be reasonable to check corectness of results presented in these references.

\section{Subsequent papers published in 2008-2012}

Notice that the first paper published in "Inverse Problems" in 2001 generated long series of wrong papers with the same mistake in the proof in the other journals with very high impact, for example:

Guozheng Yan, The problem of scattering by a partially coated crack, Nonlinear Analysis, 2008, v.68, pp. 932-939. (see wrong relations (53), (54), and Theorem 3.1 is not proved).

Guozheng Yan, The Problem of Scattering by a Mixture of Cracks and Obstacles, Boundary Value Problems, 2009, Volume 2009, Article ID 524846, 19 pages. doi:10.1155/2009/524846 (see wrong relations (3.12), and Theorems 3.1 and 3.3 are not proved). 
Guozheng Yan and Mao Yao, The method of boundary integral equations for a mixed scattering problem, Journal of Differential Equations, 2009, v. 246, pp. 4618-4631. (see wrong relation (65), and Theorem 3.1 is not proved).

Guozheng Yan and Mao Yao, Mathematical basis of scattering problems from penetrable obstacles and cracks, Journal of Mathematical Physics, 2010, v. 51, paper 123520, doi: 10.1063/1.3525831 (see wrong relations (65), (66), and Theorems 3.1 and 3.3 are not proved).

Guozheng Yan, Jianguo Ye and Jun Guo, Mathematical analysis of scattering problems for partially coated obstacles and cracks, Journal of Mathematical Physics, 2012, v. 53, paper 073702, http://dx.doi.org/10.1063/1.4731225 (see wrong relations (54), (55), and Theorem 3.1 and Lemma 3.4 are not proved).

Guozheng Yan, The method of integral equation for scattering problem with a mixed crack, Mathematical Methods in the Applied Sciences, 2011, v.34, N 15, pp. 813-821. (see wrong relations (67), (68), (69), (70), and Theorem 3.1 is not proved).

Guozheng Yan and Jianguo Ye, Boundary integral method for scattering problems with cracks buried in a piecewise homogeneous medium, Mathematical Methods in the Applied Sciences, 2012, v.35, N 1, pp. 84-96. (see wrong relations (63), (64), and Theorem 3.1 is not proved).

\section{Final remarks}

Notice that the list of mistaken papers presented in the previous section may be incomplete.

Notice also that wrong papers have been published during 11 years, and they passed reviewing and editing process in high-impact journals successfully. All these papers received positive reviews in Mathematical Reviews and in Zentralblatt MATH database.

Finally, it should be noted that the mistake in all papers is very simple and at the level of undergraduate students: authors were not able to construct adjoint integral operator correctly.

In fact, authors of the first mistaken paper discussed in section 2, are also authors of well-known books in mathematics, and they are also editorial board members of journals with high impact factor. It seems, neither Referees nor authors of subsequent papers could imagine that such people may do mistakes at the level of undergraduate students in their published papers.

\section{$5 \quad$ Reaction of editors}

Editor of Journal of Mathematical Physiscs and Editors of Nonlinear Analysis did not agree to publish a letter with explanations of mistakes. They suggested to submit a paper that should pass through process of review. However who could review the paper? The same journals reviewers, who recommended to accept three wrong papers already. It would not be reasonable to trust such Referees and such review process. In addition, construction of adjoint linear integral operator in a complex space is not a problem for reviewers, it is an exercise for 3rd year student at exams, it takes 1 line and 10 minutes. It is a pity that Editor of Journal of Mathematical Physiscs and Editors of Nonlinear Analysis did not want to find 10 minutes to do this work by theirselves. In my opinion, these Editors do not respect their readers, since they do not want to inform them about mistaken papers published in their journals.

Editors of Mathematical Methods in the Applied Sciences and Editors of Journal of Differential Equations decided to consult with each other for a while, and, finally, did not reply before submission of the present note for publication. 
Editors of Boundary Value Problems and Editor of Inverse Problems did not reply to suggestion to publish a letter with explanations of mistakes.

Probably, this is an attempt of some Editors to preserve good reputation of their journals from their point of view. However publication of a letter with explanation of mistakes is very urgent, since it may occur that many other mathematicians use mistaken method in their research at the moment, and we do not know them. So, these mathematicians should be informed as soon as possible.

In addition, acceptance of a wrong paper is a mistake of Editors, Referees, and, most likely, editorial policy of the journal, and such a mistake should be recognized.

\section{Conclusions}

People should not trust papers in high-impact journals, as well as they should not trust any other papers. Before using new results or methods in the research, they should be carefully checked.

Many readers of a present note met situation when their correct papers are rejected from high impact journals without obvious reasons. Now these readers may be sure that the same journals accept wrong papers instead of correct ones. Seems, this is a serious lack of the editorial policy of some journals, and it should be discussed in mathematical community.

In addition, seems, there is a "fatal error" in organization of so-called "independent review process" in high-impact mathematical journals, since too many different journals were involved in publishing wrong papers for a long time, and since too many times wrong papers passed through this so-called "independent review process" successfully. This "fatal error" should be corrected. The situation discussed in the present note is catastrophic for high-impact mathematical journals.

\section{References}

[1] Krutitskii P.A., "The Dirichlet problem for the dissipative Helmholtz equation in a plane domain bounded by closed and open curves", Hiroshima Mathematical Journal, Vol.28, (1998), pp.149-168. (open access).

[2] Nikiforov A. F., Uvarov V. B., Special functions of mathematical physics, Birkhauser, Basel, (1988). 